\title{
Canonical Transformations, Quantization, Mutually Unbiased and Other Complete Bases
}

\author{
Donald J. Kouri1,2, Cameron L. Williams², Nikhil Pandya ${ }^{1,2}$ \\ ${ }^{1}$ Department of Physics, University of Houston, Houston, TX, USA \\ ${ }^{2}$ Department of Mathematics, University of Houston, Houston, TX, USA \\ Email: kouri@central.uh.edu
}

How to cite this paper: Kouri, D.J., Williams, C.L. and Pandya, N. (2017) Canonical Transformations, Quantization, Mutually Unbiased and Other Complete Bases. Applied Mathematics, 8, 901-919.

https://doi.org/10.4236/am.2017.87071

Received: March 30, 2017

Accepted: July 9, 2017

Published: July 12, 2017

Copyright $\odot 2017$ by authors and Scientific Research Publishing Inc. This work is licensed under the Creative Commons Attribution International License (CC BY 4.0).

http://creativecommons.org/licenses/by/4.0/

\begin{abstract}
Using ideas based on supersymmetric quantum mechanics, we design canonical transformations of the usual position and momentum to create generalized "Cartesian-like" positions, $W$, and momenta, $p_{W}$, with unit Poisson brackets. These are quantized by the usual replacement of the classical $x, p_{x}$ by quantum operators, leading to an infinite family of potential "operator observables". However, all but one of the resulting operators are not Hermitian (formally self-adjoint) in the original position representation. Using either the chain rule or Dirac quantization, we show that the resulting operators are "quasi-Hermitian" relative to the $x$-representation and that all are Hermitian in the $W$-representation. Depending on how one treats the Jacobian of the canonical transformation in the expression for the classical momentum, $p_{W}$, quantization yields a) continuous mutually unbiased bases (MUB), b) orthogonal bases (with Dirac delta normalization), c) biorthogonal bases (with Dirac delta normalization), d) new $W$-harmonic oscillators yielding standard orthonormal bases (as functions of $W$ ) and associated coherent states and Wigner distributions. The MUB lead to $W$-generalized Fourier transform kernels whose eigenvectors are the $W$-harmonic oscillator eigenstates, with the spectrum $( \pm 1, \pm i)$, as well as "W-linear chirps". As expected, $W, p_{W}$ satisfy the uncertainty product relation: $\Delta W \Delta p_{W} \geq 1 / 2, \quad \hbar=1$.
\end{abstract}

\section{Keywords}

Canonical Transformations, Quantization, Mutually Unbiased Bases, Complete Bases

\section{Introduction}

Basis sets are of prime importance in quantum mechanics because they are fun- 
damentally connected to observables. Observables dictate the relevant operators in quantum mechanics, which are required to be self-adjoint. Some reasons underlying this are: 1) a basic postulate of quantum mechanics is that the results of measurements must be real and the eigenvalues of the relevant operator are the only possible results when measuring an observable; 2) the eigenstates of observables must be complete, spanning the relevant physical Hilbert space of possible states (as a result of the requirement that their eigenvalues are the only possible results of measurements); 3) the eigenstates can always be arranged to be mutually orthogonal (because measurements lead to an orthogonal projection of the state of the system onto an eigenstate of the observable's operator). The requirement that quantum mechanical operators for observables be self-adjoint is sufficient to ensure that the above properties hold, but one can ask "is it also necessary"? Certainly, an examination of the textbooks and most of the literature dealing with quantization of classical mechanics would suggest that it is [1]-[7]. However, interesting results have been obtained recently using non-self adjoint Hamiltonians (besides those describing meta-stable systems) [8]-[20]. In the course of these studies, attention has focused on the Hamiltonian operator and systems with discrete spectra. In this paper, we explore systematic quantizations that lead to non-self adjoint, but "quasi-Hermitian" operators [8]-[20] that potentially may describe continuous observables similar to generalized "positions" and "momenta".

In addition, complete sets of functions are also of great importance for the computational aspects of quantum mechanics. For most systems of physical interest, exact solutions are not possible and therefore approximations are the "order of the day". A great many of these rely on finding the best possible basis set to use in expanding the desired solutions (e.g., both in perturbation theory and variational calculations). It follows that one is always interested in finding new, more optimal basis sets for applications. This connection between observables and complete bases in quantum mechanics suggests that one should also seek the optimum variables (observables) to describe the system of interest.

Yet another area where basis sets play an important role is in quantum optics. In this case, coherent states (which typically are over-complete, non-orthogonal bases) are the center of focus. Especially of interest are those associated with operators that form a Lie algebra (for constructing displacement operators) or that are eigenstates of (non-self adjoint) annihilation operators.

Most recently, the field of quantum computing and quantum cryptography has generated interest in finding new groups of basis sets that are "mutually unbiased" [21] [22] [23] [24]. Although most efforts have concentrated on finite dimensional bases, there is also significant interest in discovering continuously infinite systems, the prime example of which are the (improper) eigenstates of the three self adjoint operators $\hat{x}, \hat{p}_{x}, \hat{x}+\hat{p}_{x}$. A fundamental property of $\hat{x}$ and $\hat{p}_{x}$ is that the minimum uncertainty product $\Delta x \Delta p_{x}$ is generated by the Gaussian. This result is due to the fact that the minimizing state, $\left|\psi_{\min }\right\rangle$, satisfies $\hat{x}\left|\psi_{\min }\right\rangle \propto \hat{p}_{x}\left|\psi_{\min }\right\rangle$. The MUB nature of the eigenstates of the set $\hat{x}, \hat{p}_{x}, \hat{x}+\hat{p}_{x}$ 
appears to be related to the fact that exact knowledge of $x\left(p_{x}\right)$ implies infinite uncertainty in $p_{x}(x)$. This is also related to the fact that $\left[\hat{x}, \hat{p}_{x}\right]=i \hat{1}$. One suspects that this is a characteristic required of continuous MUB [23]. Indeed, there is a number of important types of complete basis sets associated with position and momentum and they are relevant in various areas of interest. The position and momentum representations are ubiquitous in quantum mechanics. In addition, the function $\left\langle x \mid p_{x}\right\rangle=\frac{\mathrm{e}^{i k x}}{\sqrt{2 \pi}}$ is fundamental not only in quantum mechanics but also in all the sciences, engineering, signal processing, probability theory and diffusion processes, medicine, etc. In addition, the harmonic oscillator (with its symmetric quadratic dependence on the position and momentum operators) provides a complete set of eigenstates that are also eigenfunctions of the Fourier transform, and the ground state minimizes the product of the position and momentum uncertainties (variances). This uncertainty product minimizing state leads to one particular realization of coherent states (there are many others, including an infinite variety arising from application of a displacement operator (in terms of the position and momentum operators) to any normalizable "fiducial" state [25]).

In light of the role played by the position and momentum coordinates in obtaining complete bases of various types, it is natural to inquire as to the role that is played by canonical transformations of the type $x \rightarrow W, p_{x} \rightarrow p_{W}$, such that the Poisson bracket satisfies $\left\{W, p_{W}\right\}=1$. This will naturally involve how one proceeds from classical to quantum mechanics (we shall use the Dirac canonical quantization procedure). We find that canonical transformations can be used to create new, useful complete bases, which may be orthogonal, biorthogonal or MUB. We also find that the new "position" and "momentum" operators lead to generalized harmonic oscillator systems. This leads to new coherent states and generalized Fourier kernels, along with Wigner distributions. While the paper focusses on quantum mechanics, we point out the relevance of some of the results for certain classical processes.

\section{Classical Dynamical Considerations}

Our approach to the construction of new, complete sets of functions has its foundation in the concept of canonically conjugate variables and canonical transformations. In this study, we focus on point transformations [26], beginning with the standard canonically conjugate variables, $x$ and $p_{x}$. These basic variables are characterized by the property that their Poisson bracket equals one. Dirac based an approach to quantization in which the canonical variables are replaced by appropriate operators, the Poisson bracket is replaced by the commutator of the relevant operators and the scalar " 1 " is replaced by it times the identity operator [1] [7]. As is well known, the quantum mechanical operators $\hat{x}, \hat{p}_{x}$ provide the standard, continuous complete sets of orthonormal (under the Dirac delta normalization) "eigenvectors". Adding the eigenstates of the combined $\hat{x}+\hat{p}_{x}$ operator, these complete basis sets are well known to consti- 
tute a set of mutually unbiased bases (MUB) [21] [22] [23] [24]. It appears that a key component needed for this property is that the position and momentum are canonically conjugate classical variables, which when quantized, yield a constant quantum mechanical commutator. We speculate that this property is a necessary (but not sufficient) condition to obtain complete sets of eigenstates that are MUBs [23]. There is, of course, a long history of canonical transformations in classical dynamics as the means of constructing "natural" generalized, canonically conjugate coordinates in terms of which the dynamics is the simplest [26] [27] [28] [29]. Many examples exist where the natural dynamical variables are quite different from the original $x$ and $p_{x}$, and their discovery generally reflects some fundamental feature of the dynamics. In this paper, we shall explore replacing the Cartesian position and momentum by new, canonically conjugate variables and then, using Dirac quantization, explore the properties of the resulting quantum mechanical operators and their (improper) eigenstates. In the process, we shall explore both manifestly self adjoint and apparently non-self adjoint operators.

To do this, we require some guide as to possible reasonable choices for new "displacement or position variables" and their canonically conjugate momenta (once the "position-like" variable is decided upon, the conjugate momentum is determined by requiring its Poisson bracket with the new "position" be equal to 1). A hint for choosing such new variables is found in super symmetric quantum mechanics (SUSY) [30] [31] [32]. In one dimensional SUSY, the nodeless ground state for a system (in the domain $-\infty<x<\infty$ ) is expressed as

$$
\psi_{0}(x)=\psi_{0}(0) \exp \left[-\int_{0}^{x} \mathrm{~d} x^{\prime} W\left(x^{\prime}\right)\right]
$$

which has a SUSY ground state energy equal to zero. As discussed in earlier work [31], this state minimizes the uncertainty product $\Delta W \Delta p_{x}$. However, the commutator of $W$ with the standard momentum operator is proportional to $\frac{\mathrm{d} W}{\mathrm{~d} x}$, which is constant only for $W=x$ (plus an arbitrary constant that simply shifts the energy levels). Thus, in general, $p_{x}$ and $W$ are not canonically conjugate variables. Equation (1) also implies the relation

$$
V=W^{2}-\frac{\mathrm{d} W}{\mathrm{~d} x}=W^{2}+\frac{i}{\hbar}\left[\hat{W}, \hat{p}_{x}\right]
$$

between the physical potential and $W$. It is the fact that $\hat{p}_{x}$ and $\hat{W}$ are not canonically conjugate operators that is responsible for the "anharmonicity" of the system. However, this relation of W to the potential energy led the SUSY literature to designate W as a "super potential" [30]. Recent work [31] has shown that an alternate but profitable way to interpret $\mathrm{W}$ is as a "generalized position" or "displacement variable". This is reinforced by the observation that the quintessential example for $\mathrm{W}$ is that for the harmonic oscillator:

$$
W(x)=x \text {. }
$$

This suggests that the general $\mathrm{W}$ can be considered a position variable and not a potential, super or otherwise. This view has led to the development of new 
“system adapted Klauder-Skagerstam coherent states" [25] [31]. These new coherent states provide a superior basis set (compared to standard Gaussian-based coherent states or to a standard harmonic oscillator basis) for computing accurate excited state energies for some polynomial choices of $\mathrm{W}$ (which result in anharmonic oscillators). More recently, Williams, et al. [33] [34] have shown that new one dimensional, generalized harmonic oscillator systems, characterized by the $\mathrm{su}(1,1)$ Lie algebra, can be constructed. This work is based on identifying $x^{n}, n$ being an integer, as a new variable. However, this work did not develop the new variable as resulting from a canonical transformation but solely from the same SUSY ideas we are exploring here [31]. In addition, their development restricted the new variable to be a monomial function of $\mathrm{x}$.

This suggests that, in addition to introducing the requirement that the new variable result from a canonical transformation of $\mathrm{x}$, that a useful family of W's might be polynomials in $\mathrm{x}$, whose lowest and highest powers are odd, and with non-negative coefficients (to ensure monotonicity and that the domain remains $(-\infty, \infty))$. We therefore restrict ourselves to W's of the form

$$
W(x)=\sum_{j=1}^{2 J+1} a_{j} x^{j}, a_{j} \geq 0 .
$$

The coefficients are also restricted such that the coefficient of each even power is never larger than the coefficient of the next lower odd power. This is sufficient to ensure that the ground state is not only normalizable but that the $x \rightarrow W$ transformation is one-to-one and onto (it has a derivative that is non-negative so it is a monotonic function and invertible). We remark that the new variable, $W(x)$, corresponds to "stretching" the real line. Also, we interpret $W$ as a "Cartesian-like" variable in the following sense. The distance (metric) between any two points, $W_{1}$ and $W_{2}$, is $S^{2}=\left(W_{2}-W_{1}\right)^{2}$. This leads to a measure that is the square root of the differential metric. The original (classical) canonical variables are $\left(x, p_{x}\right)$. We then require that the new, canonically conjugate momentum, $p_{W}$, be a function of $\left(x, p_{x}\right)$ such that the Poisson bracket satisfies

$$
\left\{W, p_{W}\right\}=1 \text {. }
$$

This is easily solved to yield the general classical expression

$$
p_{W} \equiv \frac{1}{\left(\frac{\mathrm{d} W}{\mathrm{~d} x}\right)^{1-\alpha}} p_{x} \frac{1}{\left(\frac{\mathrm{d} W}{\mathrm{~d} x}\right)^{\alpha}}+g(x),
$$

where $g(x)$ is the constant of integration of Equation (5) along a path of constant $x$. We invoke the simplest assumption of taking the solution with $g(x)=0$. We see that the Jacobian of the transformation can be split in infinitely many ways classically since all the quantities commute. (The condition Equation (1) remains true for any choice of $\alpha$.) It is immediately clear, of course, that unless $\alpha=1 / 2$, this expression will suffer from the usual issue that when quantized by replacing the position and momentum by the standard quantum mechanical position and momentum operators, it will typically result in nonself-adjoint momentum operators in the $\mathrm{x}$-representation (with respect to the 
measure " $\mathrm{d} x$ "). A common procedure to ensure self adjointness is to define a new canonical momentum as the arithmetic average

$$
p_{W}=\frac{1}{2}\left[\frac{1}{\left(\frac{\mathrm{d} W}{\mathrm{~d} x}\right)^{1-\alpha}} p_{x} \frac{1}{\left(\frac{\mathrm{d} W}{\mathrm{~d} x}\right)^{\alpha}}+\frac{1}{\left(\frac{\mathrm{d} W}{\mathrm{~d} x}\right)^{\alpha}} p_{x} \frac{1}{\left(\frac{\mathrm{d} W}{\mathrm{~d} x}\right)^{1-\alpha}}\right] .
$$

Clearly, in the x-representation, this leads to a self-adjoint operator when quantized. We also observe that Equation (6) above yields a manifestly self-adjoint momentum operator for the choice $\alpha=1 / 2$, which is also included in Equation (7). However, all of these definitions of the classical canonical momentum yield a Poisson bracket equal to 1 . We now have the classical canonically conjugate pair $\left\{x, p_{x}\right\}$ and an infinity of possible new, canonically conjugate variables $\left\{W(x), p_{W}\right\}$. Due to the $\frac{\mathrm{d} W}{\mathrm{~d} x}$ factors, it is convenient to quantize in the $\mathrm{x}$-representation and choosing to quantize with Equation (6) or (7) will result in different quantum operators, whose (improper) eigenstates are the subject of our investigations.

\section{Dirac Quantization of the Canonically Conjugate Classical Variables}

We now consider the quantization of the new canonically conjugate variables. But there are infinitely many equally valid classical expressions for the new canonical momentum. To illustrate, consider quantizing the choices $\alpha=0,1$ for Equation (6) by replacing the classical variables by the standard quantum mechanical position and momentum operators: $\alpha=0$ :

$$
\hat{p}_{W}=\frac{-i}{\mathrm{~d} W / \mathrm{d} x} \frac{\mathrm{d}}{\mathrm{d} x}
$$

and $\alpha=1$ :

$$
\hat{p}_{W}=-i \frac{\mathrm{d}}{\mathrm{d} x} \frac{1}{\mathrm{~d} W / \mathrm{d} x} .
$$

These are clearly not self-adjoint operators in the x-representation. (Indeed, Equation (9) is the adjoint of Equation (8).) Therefore, one would normally discard both. However, we note that for all of the infinitely many definitions of the canonical momenta (including Equation (7)), their Poisson bracket with $W$ is equal to 1 (see Equation (5)). Thus, Equations (6) and (7) both yield the Dirac quantization result

$$
\left[\hat{W}, \hat{p}_{W}\right]=i \hat{1}
$$

Appealing to Occam's razor, this immediately implies that the corresponding quantum operators in the $W$-representation are

$$
\hat{W}=W, \hat{p}_{W}=-i \frac{\mathrm{d}}{\mathrm{d} W} .
$$


Interestingly, employing the chain rule for derivatives, Equation (8) directly yields $\frac{-i}{\mathrm{~d} W / \mathrm{d} x} \frac{\mathrm{d}}{\mathrm{d} x} \equiv-i \frac{\mathrm{d}}{\mathrm{d} W}$. So $\hat{W}$ and $\hat{p}_{W}$, as given in Equation (8), are manifestly self-adjoint operators in the W-representation. In fact, by Equation (8), $\hat{p}_{W}$ is a quasi-Hermitian operator [8]-[20] relative to the original $\mathrm{x}$-representation momentum operator, i.e., $\hat{p}_{x}=\frac{\mathrm{d} W}{\mathrm{~d} x} \hat{p}_{W}$ or $\mathrm{d} x \hat{p}_{x}=\mathrm{d} W \hat{p}_{W}$.

Furthermore, $\frac{\mathrm{d} W}{\mathrm{~d} x}$ is a non-negative polynomial and it ensures that the measure $\mathrm{d} W=\mathrm{d} x \frac{\mathrm{d} W}{\mathrm{~d} x}$ is the square root of the metric. Note that the measure is supplied automatically by the canonical transformation. Note further that the structure of Equation (11) is identical to the original structure for the operators $\hat{x}, \hat{p}_{x} \quad$ [23]. Therefore, the minimum uncertainty product condition,

$\Delta x \Delta p_{x}=1 / 2$, becomes $\Delta W \Delta p_{W}=\Delta W(x) \Delta W(k)=1 / 2$ for the new "position" and momentum". It is then clear that there exist complete, orthonormal (in the Dirac delta function sense) eigenstates of the three operators $\hat{W}, \hat{p}_{W}, \hat{W}+\hat{p}_{W}$ which will constitute MUB.Their structure is given by

$$
\begin{gathered}
\left\langle W^{\prime} \mid W\right\rangle=\delta\left(W^{\prime}-W\right)=\frac{\mathrm{d} W}{\mathrm{~d} x} \delta\left(W(x)-W\left(x^{\prime}\right)\right), \\
\left\langle W \mid p_{W}\right\rangle=\frac{\mathrm{e}^{i p_{W} W}}{\sqrt{2 \pi}}=\frac{\mathrm{e}^{i W(k) W(x)}}{\sqrt{2 \pi}}, \\
\left\langle W \mid W+p_{W}\right\rangle=\frac{\mathrm{e}^{i p_{W} W-i W^{2} / 2}}{\sqrt{2 \pi}}=\frac{\mathrm{e}^{i W(k) W(x)-i W(x)^{2} / 2}}{\sqrt{2 \pi}},
\end{gathered}
$$

which are a set of MUB [23]. (The second parts of Equations (12)-(14) are in the $\mathrm{x}$-representation and the second parts of Equations (13)-(14) involve requiring that $W(k)=p_{W}$. This ensures that the argument of the exponential, $p_{W} W$ is dimensionless and that the momentum spectrum is also Cartesian-like. That is, it has a Cartesian metric and the measure for integration over the momentum is $\left.\mathrm{d} k \frac{\mathrm{d} W(k)}{\mathrm{d} k}=\mathrm{d} p_{W}\right)$. Equation (14) is recognized as a linear chirp in $W$. When using the above for quantum cryptography, one can work in the original $\mathrm{x}$-coordinate representation, adding an additional layer of security because there are infinitely many choices for $W$ and each one results in a different $\mathrm{x}$-representation MUB. The measure is automatically taken into account through the chain rule expression $\mathrm{d} W=\mathrm{d} x \frac{\mathrm{d} W}{\mathrm{~d} x}$. We arrive at the result that even though all the choices, Equation (6), for the canonically conjugate momentum (except for $\alpha=1 / 2$ ) lead to non-self adjoint (quasi-Hermitian) momentum operators in the coordinate representation, by direct application of Dirac quantization to the Poisson bracket and under the assumption of greatest simplicity, they all result in the same self-adjoint "position" and "momentum" operators in the W-representation. This is also true for the self-adjoint choices, Equation (7), and therefore all choices lead to an unique MUB for each possible $W$. We next explore the 
interesting issue of self-adjointness, and its lack, in the coordinate representation of the new operators.

\section{Lack of Self-Adjoint Property for Canonical Variables Not Quantized by Dirac's Method}

We can quantize the infinite set of canonically conjugate variables that result in non-self adjoint operators in the coordinate representation (along with those that do result in self-adjoint quantum operators, but, as we shall see, do not directly yield MUB in the coordinate representation). It is easily shown that the commutator of $\hat{W}$ and any of the possible coordinate representation "momentum operators" equals $i \hbar \hat{1}$. For example, as Equation (8) stands, it clearly results in a momentum operator which is not self-adjoint in the x-representation. However, as discussed above, based on the "chain rule" for derivatives, it has the precise, self-adjoint form

$$
\hat{p}_{W}=-i \frac{\mathrm{d}}{\mathrm{d} W}
$$

in the $\mathrm{W}$-representation. Thus, although Equation (8) is not self-adjoint in the $\mathrm{x}$ representation, it is in the $\mathrm{W}$-representation. In fact, this is true for all the possible choices of $p_{W}$, provided that one assumes that the only sensible quantization of $W$ in the $W$-representation is $\hat{W}=W$ and this, along with Equation (10) and Occam's razor, implies Equation (15). This is evidently self-adjoint, but only with respect to the measure $d W$. This shows that Equation (8) is a valid way to quantize systems such that one obtains a self-adjoint momentum operator without symmetrizing (albeit with a new measure). It is instructive to consider the self-adjoint property of Equation (11) in more detail. It is clear that

$$
\int_{-\infty}^{\infty} \mathrm{d} W \psi^{*}(W)\left[-i \frac{\mathrm{d}}{\mathrm{d} W}\right] \phi(W)=\int_{-\infty}^{\infty} \mathrm{d} W \phi(W)\left[-i \frac{\mathrm{d}}{\mathrm{d} W}\right] \psi^{*}(W) .
$$

In the $\mathrm{x}$-representation, this equals

$$
\begin{gathered}
\int_{-\infty}^{\infty} \mathrm{d} x \frac{\mathrm{d} W}{\mathrm{~d} x} \tilde{\psi}^{*}(x)\left[\frac{-i}{\mathrm{~d} W / \mathrm{d} x} \frac{\mathrm{d}}{\mathrm{d} x}\right] \tilde{\phi}(x)=\int_{-\infty}^{\infty} \mathrm{d} x \tilde{\psi}^{*}(x)\left[-i \frac{\mathrm{d}}{\mathrm{d} x}\right] \tilde{\phi}(x) \\
=\int_{-\infty}^{\infty} \mathrm{d} x \tilde{\phi}(x)\left[-i \frac{\mathrm{d}}{\mathrm{d} x}\right] \tilde{\psi}^{*}(x) \\
\equiv \int_{-\infty}^{\infty} \mathrm{d} x \frac{\mathrm{d} W}{\mathrm{~d} x} \tilde{\phi}(x)\left[\frac{-i}{\mathrm{~d} W / \mathrm{d} x} \frac{\mathrm{d}}{\mathrm{d} x}\right] \tilde{\psi}^{*}(x) .
\end{gathered}
$$

Thus, we see explicitly that Equation (8) is self-adjoint under a measure which we again stress is automatically dictated by the canonical transformation. This suggests that we explore the x-representation adjoints of other choices for the canonical momentum. To do this, we treat the general expression, Equation (6) since it includes all the obvious ways to split the Jacobian factor. Of course, the symmetrized expression, Equation (7) is obviously self-adjoint in the coordinate representation. We consider the integral 


$$
\int_{-\infty}^{\infty} \mathrm{d} x \phi^{*}(x) \frac{-i}{\left(\frac{\mathrm{d} W}{\mathrm{~d} x}\right)^{1-\alpha}} \frac{\mathrm{d}}{\mathrm{d} x} \frac{1}{\left(\frac{\mathrm{d} W}{\mathrm{~d} x}\right)^{\alpha}} \psi(x) .
$$

We define

$$
\begin{gathered}
\bar{\phi}^{*}=\frac{\phi^{*}}{\left(\frac{\mathrm{d} W}{\mathrm{~d} x}\right)^{1-\alpha}}, \\
\bar{\psi}=\frac{\psi}{\left(\frac{\mathrm{d} W}{\mathrm{~d} x}\right)^{\alpha}},
\end{gathered}
$$

and it immediately follows that

$$
\int_{-\infty}^{\infty} \mathrm{d} x \psi(x) \frac{-i}{\left(\frac{\mathrm{d} W}{\mathrm{~d} x}\right)^{\alpha}} \frac{\mathrm{d}}{\mathrm{d} x} \frac{1}{\left(\frac{\mathrm{d} W}{\mathrm{~d} x}\right)^{1-\alpha}} \phi^{*}(x) .
$$

It is obvious that unless $\alpha=1 / 2$, the momentum operator is not self-adjoint in the $\mathrm{x}$-representation. This is related to the role of the measure, which arises due to the Jacobian of the canonical transformation.

Our next consideration is to determine whether the operators in Equations (6)-(7), are self-adjoint under the measure $\mathrm{d} x \frac{\mathrm{d} W}{\mathrm{~d} x}$. We only need to treat Equation (6) directly since the results will dictate those for Equation (7). We thus consider the integral

$$
\begin{aligned}
& I_{\alpha}=\int_{-\infty}^{\infty} \mathrm{d} x\left(\frac{\mathrm{d} W}{\mathrm{~d} x}\right) \psi^{*}(x) \frac{1}{\left(\frac{\mathrm{d} W}{\mathrm{~d} x}\right)^{1-\alpha}} \hat{p}_{x} \frac{1}{\left(\frac{\mathrm{d} W}{\mathrm{~d} x}\right)^{\alpha}} \phi(x) \\
&=\int_{-\infty}^{\infty} \mathrm{d} x \psi^{*}(x) \frac{1}{\left(\frac{\mathrm{d} W}{\mathrm{~d} x}\right)^{-\alpha}} \hat{p}_{x} \frac{1}{\left(\frac{\mathrm{d} W}{\mathrm{~d} x}\right)^{\alpha}} \phi(x) \\
&=\int_{-\infty}^{\infty} \mathrm{d} x \phi(x) \frac{1}{\left(\frac{\mathrm{d} W}{\mathrm{~d} x}\right)^{\alpha}} \hat{p}_{x} \frac{1}{\left(\frac{\mathrm{d} W}{\mathrm{~d} x}\right)^{-\alpha}} \psi^{*}(x) \\
&= \int_{-\infty}^{\infty} \mathrm{d} x\left(\frac{\mathrm{d} W}{\mathrm{~d} x}\right) \phi(x) \frac{1}{\left(\frac{\mathrm{d} W}{\mathrm{~d} x}\right)^{1+\alpha}} \hat{p}_{x} \frac{1}{\left(\frac{\mathrm{d} W}{\mathrm{~d} x}\right)^{-\alpha}} \psi^{*}(x)
\end{aligned}
$$

This shows that unless $\alpha=0, \hat{p}_{W}$ in Equation (6) is not self-adjoint under the measure $\mathrm{d} W=\mathrm{d} x\left(\frac{\mathrm{d} W}{\mathrm{~d} x}\right)$. We summarize:

(1) For Equation (6) with $\alpha=1 / 2$ and Equation (7), $\hat{p}_{W}$ is self-adjoint under the measure $\mathrm{d} x$ (i.e., in the $\mathrm{x}$-representation).

(2) For $\alpha \neq 1 / 2$, Equation (6) is not self-adjoint under the measure $\mathrm{d} x$ (i.e., in the $\mathrm{x}$-representation).

(3) For $\alpha=0$, Equation (6) is self-adjoint under the measure $\mathrm{d} W$ (i.e., in the 
W-representation).

(4) For $\alpha \neq 0$, Equation (6) (and also clearly for Equation (7)) is not self-adjoint in the $\mathrm{W}$-domain.

(5) For $\alpha=0$, Equation (6) gives rise to continuous MUB for the operators $\hat{W},-i \frac{\mathrm{d}}{\mathrm{d} W}, \hat{W}-i \frac{\mathrm{d}}{\mathrm{d} W}$.

We next show that the role of the measure is to ensure that these non-self adjoint (as well as the self-adjoint) choices of momentum operators give rise to complete, continuous $\mathrm{x}$-representation, biorthogonal (or orthogonal in the selfadjoint cases) basis sets with Dirac delta normalization.

\section{Continuous Biorthogonal Basis Sets for Canonically Conjugate, Non-Self Adjoint Operators in the $\mathrm{x}$-Representation}

The key to understanding the role of the Jacobian and measure for the improper eigenstates of the new, non-self adjoint momentum operators is to evaluate them explicitly in the coordinate representation. We treat the general case of Equation (6). Thus, we solve the eigenequation

$$
\left[\frac{-i}{\left(\frac{\mathrm{d} W}{\mathrm{~d} x}\right)^{1-\alpha}} \frac{\mathrm{d}}{\mathrm{d} x} \frac{1}{\left(\frac{\mathrm{d} W}{\mathrm{~d} x}\right)^{\alpha}}\right] \phi_{p_{W}}(x)=p_{W} \phi_{p_{W}}(x)
$$

where $p_{W}$ is the eigenvalue. We recognize that, in general, the above momentum operator $(\alpha \neq 1 / 2)$ is not self-adjoint in the $\mathrm{x}$-representation. However, it turns out that when one considers complex eigenvalues, $p_{W}$, the new states are not normalizable, even in the Dirac delta sense. As a result, we need only consider real eigenvalues for Equation (28). It is easily shown that the solution is

$$
\left\langle x \mid \phi_{p_{W}}\right\rangle=\phi_{p_{W}}(x)=\frac{\left(\frac{\mathrm{d} W}{\mathrm{~d} x}\right)^{\alpha}}{\sqrt{2 \pi}} \mathrm{e}^{i W(k) W(x)} .
$$

Here, we again replace the eigenvalue $p_{W}$ by $W(k)$ since the argument of the exponential must be dimensionless and symmetric. In similar fashion, we also easily find that the dual eigenvector is

$$
\left\langle\phi_{p_{W}} \mid x\right\rangle=\phi_{p_{W}}^{*}(x) \frac{\left(\frac{\mathrm{d} W}{\mathrm{~d} x}\right)^{1-\alpha}}{\sqrt{2 \pi}} \mathrm{e}^{i W(k) W(x)} .
$$

We then see that the eigenstates satisfy the completeness relation

$$
\left\langle\phi_{\bar{p}_{W}} \mid \phi_{p_{W}}\right\rangle=\frac{1}{2 \pi} \int_{-\infty}^{\infty} \mathrm{d} x\left(\frac{\mathrm{d} W}{\mathrm{~d} x}\right)^{1-\alpha+\alpha} \mathrm{e}^{i\left(p_{W}-\bar{p}_{W}\right) W(x)}=\delta\left(\bar{p}_{W}-p_{W}\right) .
$$

Thus, the eigenstates and their duals automatically supply the required factors to produce the correct measure, leading to a complete biorthogonal (or orthogonal when $\alpha=1 / 2$ ) basis. 
This is analogous to a situation previously studied by Kouri, et al. [15] where they showed that the harmonic oscillator Hamiltonian could be transformed similarly to a non-self adjoint form, leading to eigenstates that belong to a biorthogonal complete set. It is also clear that these bases will not be part of any MUB because there is always a non-constant-modular factor in the eigenvector and/or its dual. Furthermore, even for the self-adjoint choice $\alpha=1 / 2$, there will not be an MUB. In particular, we also see that neither of the choices $\alpha=0$ or $\alpha=1$ leads to an MUB in the coordinate representation. It follows from this that the same is true for the other self-adjoint choice, Equation (7).

We now determine the x-representation eigenstates of Equation (7) (which includes Equation (6) for $\alpha=1 / 2$ ) for the general, self-adjoint $\hat{p}_{W}$. We consider

$$
\frac{1}{2}\left[\frac{1}{\left(\frac{\mathrm{d} W}{\mathrm{~d} x}\right)^{1-\alpha}} \hat{p}_{x} \frac{1}{\left(\frac{\mathrm{d} W}{\mathrm{~d} x}\right)^{\alpha}}+\frac{1}{\left(\frac{\mathrm{d} W}{\mathrm{~d} x}\right)^{\alpha}} \hat{p}_{x} \frac{1}{\left(\frac{\mathrm{d} W}{\mathrm{~d} x}\right)^{1-\alpha}}\right] \phi_{p_{W}}(x)=p_{W} \phi_{p_{W}}(x) .
$$

This is easily seen to give

$$
\frac{-i}{\left(\frac{\mathrm{d} W}{\mathrm{~d} x}\right)} \frac{\mathrm{d} \phi_{p_{W}}}{\mathrm{~d} x}-\frac{i}{2} \phi_{p_{W}} \frac{\mathrm{d}}{\mathrm{d} x} \frac{1}{\left(\frac{\mathrm{d} W}{\mathrm{~d} x}\right)}=p_{W} \phi_{p_{W}}(x) .
$$

But

$$
\frac{\mathrm{d}}{\mathrm{d} x}\left(\frac{\mathrm{d} W}{\mathrm{~d} x}\right)^{-1}=-\frac{\mathrm{d}^{2} W}{\mathrm{~d} x^{2}}\left(\frac{\mathrm{d} W}{\mathrm{~d} x}\right)^{-2},
$$

yielding the $\alpha$-independent result

$$
\frac{-i}{\left(\frac{\mathrm{d} W}{\mathrm{~d} x}\right)} \frac{\mathrm{d} \phi_{p_{W}}}{\mathrm{~d} x}+\frac{i}{2} \phi_{p_{W}} \frac{\mathrm{d}^{2} W}{\mathrm{~d} x^{2}}\left(\frac{\mathrm{d} W}{\mathrm{~d} x}\right)^{-2}=p_{W} \phi_{p_{W}}(x) .
$$

The operator $\frac{-i}{\left(\frac{\mathrm{d} W}{\mathrm{~d} x}\right)} \frac{\mathrm{d}}{\mathrm{d} x}+\frac{i}{2} \frac{\mathrm{d}^{2} W}{\mathrm{~d} x^{2}}\left(\frac{\mathrm{d} W}{\mathrm{~d} x}\right)^{-2}$ is self-adjoint under the measure " $\mathrm{d} x$ ". Equation (35) is readily integrated and yields the final result

$$
\phi_{P_{W}}(x)=\phi_{P_{W}}(0)\left(\frac{\mathrm{d} W}{\mathrm{~d} x}\right)^{1 / 2} \mathrm{e}^{i p_{W} W}=\phi_{P_{W}}(0)\left(\frac{\mathrm{d} W}{\mathrm{~d} x}\right)^{1 / 2} \mathrm{e}^{i W(k) W(x)} .
$$

This is identical, of course, to the result, Equation (30) for the special, selfadjoint choice $\alpha=1 / 2$. It is easily seen that the proper normalization is the usual $\phi_{p_{W}}(0)=\sqrt{1 / 2 \pi}$. This demonstrates that the eigenstates of all of the selfadjoint $\hat{p}_{W}$ are identical, independent of the choice of $\alpha$.

\section{Non-unitary Transformations and the New Momentum Operators}

We recognize that one can always generate MUBs by unitary transformations of existing MUBs. However, this doesn't lead to new MUBs in the present sense. 
We stress here our new MUBs are not the result of unitary transformations. This is clear from the fact that we generate non-self adjoint momentum operators. For example, the fact that in Equation (8), the new momentum operator results from a one-sided application of the operator $\frac{1}{\left(\frac{\mathrm{d} W}{\mathrm{~d} x}\right)}$ to the $\mathrm{x}$-representation momentum operator is new and opens up interesting possibilities. Indeed, in Equation (6), we again see that the transformation from the old to the new momentum operator does not correspond to a standard transformation in quantum mechanics. In addition, all the $\alpha$-dependent momentum operators can be related to that of Equation (8) via similarity transformations. We define the general similarity transform

$$
\hat{S}=\left(\frac{\mathrm{d} W}{\mathrm{~d} x}\right)^{-\alpha}, \hat{S}^{-1}=\left(\frac{\mathrm{d} W}{\mathrm{~d} x}\right)^{\alpha}
$$

Then it is easily seen that

$$
\frac{-i}{\left(\frac{\mathrm{d} W}{\mathrm{~d} x}\right)} \frac{\mathrm{d}}{\mathrm{d} x}=\hat{S} \frac{-i}{\left(\frac{\mathrm{d} W}{\mathrm{~d} x}\right)^{1-\alpha}} \frac{\mathrm{d}}{\mathrm{d} x} \frac{1}{\left(\frac{\mathrm{d} W}{\mathrm{~d} x}\right)^{\alpha}} \hat{S}^{-1} .
$$

Thus, all the possible expressions, Equation (6) (and Equation (7), since by Equation (35), it is independent of $\alpha$ ), are equivalent, under a similarity transformation, to Equation (8), which is self-adjoint under the measure

$\mathrm{d} W=\mathrm{d} x\left(\frac{\mathrm{d} W}{\mathrm{~d} x}\right)$. The measure is automatically generated by the canonical trans formation. We also point out that one could have chosen the constant of integration, $g(x)$ in Equation (6) to ensure that one obtains Equation (8), independent of the choice of $\alpha$.

\section{Generalized Harmonic Oscillators, Generalized Coherent States and Generalized Fourier Transforms}

In this Section, we point out that the operators $\hat{W}, \hat{p}_{W}$ can also be used to define a generalized harmonic oscillator with Hamiltonian

$$
\hat{H}_{W}=\frac{1}{2}\left(\hat{p}_{W}^{2}+\hat{W}^{2}\right),
$$

with $\hat{W}, \hat{p}_{W}$ given by Equation (11). The eigenstates are given by

$$
\psi_{j}(W)=c_{j} H_{j}(W) \exp \left[-\frac{W^{2}}{2}\right]
$$

where the coefficients $c_{j}$ are the usual normalization constants and the functions $H_{j}(W)$ are standard Hermite polynomials of the variable W. They are a complete, orthonormal basis, so that

$$
\sum_{j=0}^{\infty} \psi_{j}^{*}(W) \psi_{j}\left(W^{\prime}\right)=\delta\left(W-W^{\prime}\right)
$$

and 


$$
\int_{-\infty}^{\infty} \mathrm{d} W \psi_{j}^{*}\left(W^{\prime}\right) \psi_{j^{\prime}}\left(W^{\prime}\right)=\delta_{i j^{\prime}} \cdot
$$

Just as in the case of the standard harmonic oscillator, where $\Delta x \Delta k=1 / 2$ for the ground state, the $\mathrm{W}$-harmonic oscillator ground state for a given polynomial $\mathrm{W}(\mathrm{x})$ will satisfy $\Delta W(x) \Delta W(k)=1 / 2$. Next we note that Equation (39) can be factored in terms of the ladder operators

$$
\begin{gathered}
\hat{a}_{W}=\frac{1}{\sqrt{2}}\left(\frac{\mathrm{d}}{\mathrm{d} W}+W\right) \\
\hat{a}_{W}^{+}=\frac{1}{\sqrt{2}}\left(-\frac{\mathrm{d}}{\mathrm{d} W}+W\right) .
\end{gathered}
$$

Thus,

$$
\begin{aligned}
\hat{H}_{W} & =\hat{a}_{W}^{+} \hat{a}_{W}+1 / 2 \\
& =\hat{a}_{W} \hat{a}_{W}^{+}-1 / 2 .
\end{aligned}
$$

It follows that one can construct coherent states using the W-displacement operator or as eigenvectors of the lowering operator. The resulting coherent states are over-complete and can be used as basis-functions for a variety of calculations. They also can be used to explore new types of semiclassical approximations. In addition, one can generate $\mathrm{W}$-generalized Wigner distributions in the $W, P_{W}$ phase space. As for the new MUB, these all may be implemented in the coordinate representation with the correct measure guaranteed by the canonical transformation. We note that the Heisenberg-Weyl Lie algebra governs the W-oscillator, in contrast to the work of Williams, et al. [34].

Of course, the orthonormal eigenstates of the W-harmonic oscillator are eigenstates of the $\mathrm{W}$-momentum eigenstates (interpreted as generalized Fourier transform kernels). That is, the $\psi_{j}\left(W^{\prime}\right)$ satisfy the generalized Fourier transform relation

$$
\begin{aligned}
& \left\langle\phi_{p_{W}}\left|\int_{-\infty}^{\infty} \mathrm{d} W\right| W\right\rangle\left\langle W \mid \psi_{j}\right\rangle \\
& =\frac{1}{\sqrt{2 \pi}} \int_{-\infty}^{\infty} \mathrm{d} W \mathrm{e}^{-i p_{W} W} \psi_{j}(W)=\psi_{j}\left(p_{W}\right) .
\end{aligned}
$$

In the coordinate representation, this is

$$
\frac{1}{\sqrt{2 \pi}} \int_{-\infty}^{\infty} \mathrm{d} x \frac{\mathrm{d} W}{\mathrm{~d} x} \mathrm{e}^{-i W(k) W(x)} \psi_{j}(W(x))=\psi_{j}(W(k)),
$$

and it is in this form that the transformation will be used. We also note that time-frequency analyses can be carried out by a windowed generalized Fourier transform, with the window being the ground state of the W-harmonic oscillator, or any other convenient window. Such transformations should be of interest for the analysis of chirps.

Finally, we note that one can also use the W-Gaussian to generate new "minimum uncertainty wavelets" and the closely related "Hermite Distributed Approximating Functionals" that have proved to be extremely useful computational tools in a number of areas, as well as for digital signal processing [35]-[41]. 


\section{Results and Discussion}

For invertible canonical transformations of $x, p_{x}$ to Cartesian-like variables $W, p_{W}$, Dirac quantization results in the unique operators $\hat{W}=W, \hat{p}_{W}=-i \frac{\mathrm{d}}{\mathrm{d} W}$. Additionally, simple replacement of the usual position and momentum variables by $\hat{x}=x, \hat{p}=-i \frac{\mathrm{d}}{\mathrm{d} x}$ in any of the infinitely many classical expressions for $W, p_{W}$ typically leads to non-Hermitian operators. These are normally rejected as valid operators but they can all be transformed to the $\mathrm{W}$-representation Hermitian operators, $\hat{W}, \hat{p}_{W}$. The non-Hermitian x-representation operators yield biorthogonal, complete basis sets and the Hermitian cases yield a unique orthonormal complete basis set (all with Dirac delta normalization). These nonHermitian operators all are examples of quasi-Hermitian operators.

We've shown that possessing the canonical commutation relation corresponding to a unit Poisson bracket is not sufficient [23] to produce MUB. The standard method of defining a self-adjoint momentum operator as the average of the corresponding classical expressions satisfies the correct commutation relation but does not lead directly to MUBs. Neither does the symmetric or asymmetric quantization of the canonical momentum as defined in Equation (6), directly yield an MUB in the coordinate representation. Rather, it is only in the new, W-representation that one obtains an MUB [23]. In addition, for any given choice of W, this MUB is unique (as seen when expressed in the x-representation) and independent of the particular way in which one arranges the canonical momentum prior to quantization!

In the above study, we restricted ourselves to odd power dominated, nonnegative coefficient polynomial choices of the generalized position. We ask now what happens if we choose only the even powered polynomials. In that case, the eigenstates of $\hat{p}_{W}$ have exactly the same form as before. However, there are now additional, standard, normalizable eigenstates in the $L^{2}$ sense for complex eigenvalues $p_{W}=p_{W}^{\text {real }}+i p_{W}^{\text {imag }}=W^{\text {real }}(k)+i W^{\text {imag }}(k)$, of the form

$$
\begin{gathered}
\varphi_{W}(x)=\exp \left[i W^{\text {real }}(k) W(x)-W^{\text {imag }}(k) W(x)\right], \\
W(x) \stackrel{|x| \rightarrow \infty}{\longrightarrow}+\infty, W^{\text {imag }}(k)>0 .
\end{gathered}
$$

These tend to zero as $x \rightarrow \pm \infty$ and so the eigenstate structure is more complicated. In addition, their modulus is not constant and eigenstates for different eigenvalues are not orthogonal. A third important issue is the fact that the domain of $\mathrm{W}$ with even powers of $\mathrm{x}$ is $0 \leq W<\infty$. Thus, the new variable is not "Cartesian-like", since one no longer has the full real line as the domain of the new variable but only the half line. The transform is also no longer invertible except on the half line, $0 \leq x<\infty$. This is suggestive of a "radial-like" behavior, and is a situation which will be studied further, along with more general choices of $W$ (e.g., fractional powers of $x$ and others).

We also see that it is possible to obtain new, unexpected self-adjoint operators by properly accounting for the Jacobian of the canonical transformation. This 
avoids forcing self-adjointness by symetrization techniques by taking advantage of the fact that the chain rule automatically ensures that one particular ordering of the classical variables, Equation (8), is manifestly self-adjoint, with the proper measure. There is a sense in which this is an obvious point (it is well known that there are operators that are self-adjoint under one measure and not another). The point is that the change in measure is a natural consequence of our seeking to find more natural coordinates to describe the systems of interest. We stress that this has already lead us in previous studies to develop new coherent states whose convergence properties for excited states are superior to bases that are not defined using information about the ground state [31]. Our strategy here is simply to use the same ideas that make canonical transformations so useful in classical dynamics for the quantum mechanical case. The result is that we now have an infinite number of $\mathrm{W}$ choices resulting in sets of operators whose eigenstates form continuous MUBs, as well as continuous, complete biorthogonal and orthogonal basis sets and over-complete coherent states!

We constructed four distinct types of bases. These include the MUB generated by the three operators, $\left\{\hat{W}, \hat{p}_{W}, \hat{W}+\hat{p}_{W}\right\}$. Because these can be implemented in the $\mathrm{x}$-representation (with the new measure automatically taken into account), these new MUB make possible an added layer of security in applications to quantum cryptography. Next we have used the W-representation "position" and "momentum" to define the Hamiltonian of a generalized harmonic oscillator, resulting in the $\mathrm{W}$-representation orthonormal basis functions. Again, these will typically be employed in the $\mathrm{x}$-representation. We have generated new continuous, complete biorthogonal and orthogonal bases using the x-representation of the various $\hat{W}, \hat{p}_{W}$ operators. This is despite the fact that the relevant operators are not self adjoint in the $\mathrm{x}$-representation. We also have used the fact that the $\mathrm{W}$-representation harmonic oscillator Hamiltonian can be factored into the corresponding raising and lowering operators to generate new coherent states. Additionally, we also obtain new generalized Wigner distributions based on the $\mathrm{W}$-HO ground states. We note that the eigenstates of $\hat{p}_{W}$ (in the x-representation) generalize the Fourier transform, so that we have new tools (including new windowed, non-linear transforms) to carry out signal processing of non-linear, non-stationary time-frequency signals (e.g., chirps) that are not amenable to the standard Fourier transform. The eigenstates of $\hat{W}+\hat{p}_{W}$ (Equation (14)) are clearly linear chirps in the variable $\mathrm{W}$ (and therefore highly non-linear chirps when expressed as functions of $\mathrm{x}$ ).

It is perhaps useful to illustrate the robustness of one realization of the MUB in terms of a sparseness of representation condition. The prime example against which we compare is the continuous MUB arising from the operator set $\left\{\hat{x}, \hat{p}_{x}, \hat{x}+\hat{p}_{x}\right\}$. In this case, we deal with the Fourier transform and recognize that the Gaussian, $\exp \left[-x^{2} / 2\right]$, is invariant under it. Other functions that are narrower than the Gaussian (e.g., $\exp \left[-x^{6} / 2\right]$, corresponding (roughly) to the SUSY definition $W(x)=x^{5}$ or precisely to the W-harmonic oscillator choice of $\left.W(x)=x^{3}\right)$ in the $x$-representation have much slower decay than the Gaussian 
after Fourier transforming to the k-representation. This means that their Fourier $\mathrm{k}$-domain representation is not sparse. Thus, the Fourier transform is the optimum basis for representing the Gaussian; it gives the sparsest representation possible for that function but not for others. In like manner, the $\mathrm{W}$-harmonic oscillator choice of $W=x^{3}$ leads to $\exp \left[-x^{6} / 2\right]$, which is invariant under the generalized Fourier transform kernel $\phi_{p_{W}}=\frac{\mathrm{e}^{-i k^{3} x^{3}}}{\sqrt{2 \pi}}$. Thus, as expected, we have obtained the optimum (sparsest) basis for describing a system having the ground state $\exp \left[-x^{6} / 2\right]$. In addition, we also expect this generalized Fourier transform will be well suited for describing other states that are characterized by the same "coordinate" W(x) [31].

\section{Conclusion}

Canonically conjugate transformations lead automatically to quasi-Hermitian operators, and in the form of Equation (8), the new momentum operator is automatically self-adjoint in the new W-representation. Thus, no symmetrization is required to obtain the valid operator observables, $\hat{W}, \hat{p}_{W}$ and $\hat{W}+\hat{p}_{W}$. The Jacobian of the canonical transformation automatically supplies the required measure for self-adjointness. The uniqueness of the self-adjoint $\hat{W}, \hat{p}_{W}$ operators follows from the facts that: a) Dirac quantization of the Poisson bracket gives the same result independent of how the classical momentum is defined and b) the form, Equation (8), is "universal" independent of how the classical momentum is defined since all result from a similarity transformation of Equation (8). Finally, canonical quantization is justified because the canonical transformation is constructed to ensure a Cartesian metric for the new position. Additionally, the spectrum of $\hat{p}_{W}$ is structured identically to that of $\hat{W}$. We call such variables "Cartesian-like". Finally, canonical transformations lead to a rich structure of new, complete bases, generalized harmonic oscillators and Fourier kernels.

\section{Acknowledgements}

The author D. J. K. also gratefully acknowledges partial support of this research from The Fritz Haber Research Center for Molecular Dynamics of the Hebrew University of Jerusalem from 6/16 through 7/16, under the auspices of Professor R. Baer. Extensive discussions about Mutually Unbiased Bases with H. S. Eisenberg are gratefully acknowledged. Several helpful comments on the manuscript by J. Klauder are also gratefully acknowledged.

\section{Fund}

This research was supported in part under R. A. Welch Foundation Grant E0608 .

\section{References}

[1] Dirac, P.A.M. (1958) Quantum Mechanics. Oxford University Press, New York.

[2] Shankar, R. (2008) Principles of Quantum Mechanics. Springer, New York. 
[3] Commins, E.D. (2014) Quantum Mechanics: An Experimentalist's Approach. Cambridge University Press, New York.

[4] Weinberg, S. (1995) The Quantum Theory of Fields. Cambridge University Press, New York. https://doi.org/10.1017/CBO9781139644167

[5] Rusov, V.D., Vlasenko, D.S. and Mavrodiev, S.C. (2011) Quantization in Classical Mechanics and Its Relation to the Bohmian $\psi$-Field. Annals of Physics, 326, 1807. https://doi.org/10.1016/j.aop.2011.04.012

[6] Reginnato, M. (2005) Exact Uncertainty Principle and Quantization: Implications for the Gravitational Field. Brazilian Journal of Physics, 35, 476.

https://doi.org/10.1590/s0103-97332005000300018

[7] Dirac, P.A.M. (1933) The Lagrangian in Quantum Mechanics. Physikalisches Zeit. Der Sowjetunion, 3, 64.

[8] Bender, C.M. (2007) Making Sense of Non-Hermitian Hamiltonians. Reports on Progress in Physics, 70, 947. https://doi.org/10.1088/0034-4885/70/6/R03

[9] Scholtz, F.G., Geyer, H.B. and Hahne, F.J.W. (1992) Quasi-Hermitian Operators in Quantum Mechanics and the Variational Principle. Annals of Physics, 213, 74. https://doi.org/10.1016/0003-4916(92)90284-S

[10] de Gosson, M.A. (2014) Born-Jordan Quantization and the Equivalence of the Schrödinger and Heisenberg pictures. Foundations of Physics, 44, 1096. https://doi.org/10.1007/s10701-014-9831-Z

[11] Moiseyev, N. (1998) Quantum Theory of Resonances: Calculating Energies, Widths and Cross Sections by Complex Scaling. Physics Reports, 302, 212-293.

[12] Bender, C.M. and Boettcher, S. (1998) Real Spectra in Non-Hermitian Hamiltonians Having PT Symmetry. Physical Review Letters, 80, 5243. https://doi.org/10.1103/PhysRevLett.80.5243

[13] Bender, C.M., Brody, D.C. and Jones, H.F. (2002) Complex Extension of Quantum Mechanics. Physical Review Letters, 89, Article ID: 270401. https://doi.org/10.1103/PhysRevLett.89.270401

[14] Mostafazadeh, A. (2002) Pseudo-Hermiticity versus PT-Symmetry III. Journal of Mathematical Physics, 43, 3944. https://doi.org/10.1063/1.1489072

[15] Kouri, D.J., Papadakis, M., Kakadiaris, I. and Hoffman, D.K. (2003) Properties of Minimum Uncertainty Wavelets and Their Relations to the Harmonic Oscillator and the Coherent States. The Journal of Physical Chemistry A, 107, 7318-7327. https://doi.org/10.1021/jp030686s

[16] Curtright, T. and Mezincescu, L. (2007) Biorthogonal Quantum Systems. Journal of Mathematical Physics, 48, Article ID: 092106. https://doi.org/10.1063/1.2196243

[17] Geyer, H.B., Heiss W.D. and Scholtz, F.G. (2008) The Physical Interpretation of Non-Hermitian Hamiltonians and Other Observables. Canadian Journal of Physics, 86, 1195 .

[18] Mostafazadeh, A. (2010) Pseudo-Hermitian Representation of Quantum Mechanics. International Journal of Geometric Methods in Modern Physics, 7, 1191. https://doi.org/10.1142/S0219887810004816

[19] Mostafazadeh, A. (2013) Pseudo-Hermitian Quantum Mechanics with Unbounded Metric Operators. Philosophical Transactions of the Royal Society of London A, 371, Article ID: 201220050. https://doi.org/10.1098/rsta.2012.0050

[20] Brody, D.C. (2014) Biorthogonal Quantum Mechanics. Journal of Physics A: Mathematical and Theoretical, 47, Article ID: 035305. https://doi.org/10.1088/1751-8113/47/3/035305

[21] Weigert, S. and Wilkinson, M. (2008) Mutually Unbiased Bases for Continuous Va- 
riables. Physical Review A, 78, Article ID: 020303.

https://doi.org/10.1103/PhysRevA.78.020303

[22] Paul, E.C., Tasca, D.S., Rudnicki, L. and Walborn, S.P. (2016) Detecting Entanglement of Continuous Variables with Three Mutually Unbiased Bases. Physical Review A, 94, Article ID: 012303. https://doi.org/10.1103/PhysRevA.94.012303

[23] Durt, T., Englert, B.-G., Bengtsson, I. and Zyczkowski, K. (2010) On Mutually Unbiased Bases. International Journal of Quantum Information, 8, 535. https://doi.org/10.1142/S0219749910006502

[24] Nielsen, M. and Chuang, I. (2000) Quantum Computing and Quantum Information. Cambridge University Press, Cambridge.

[25] Klauder, J.R. and Skagerstam, B.-S. (1985) Coherent States: Applications in Physics and Mathematical Physics. World Scientific, Singapore. https://doi.org/10.1142/0096

[26] Goldstein, H. (1959) Classical Mechanics. Addison-Wesley, Reading, MA.

[27] Moshinsky, M. (1973) Canonical Transformations and Quantum Mechanics. SIAM Journal on Applied Mathematics, 25, 193-212. https://doi.org/10.1137/0125024

[28] Chow, T.L. (1997) Gauge Transformations Are Canonical Transformations. European Journal of Physics, 18, 467. https://doi.org/10.1088/0143-0807/18/6/013

[29] de Castro, A.E. (1999) Point Transformations Are Canonical Transformations. European Journal of Physics, 20, L11. https://doi.org/10.1088/0143-0807/20/1/007

[30] Cooper, F., Khare, A. and Sukhatme, U. (1995) Supersymmetry in Quantum Mechanics. Physics Reports, 251, 267.

[31] Chou, C.-C., Biamonte, M.T., Bodmann, B.G. and Kouri, D.J. (2012) New System-Specific Coherent States for Bound State Calculations. Journal of Physics A: Mathematical and Theoretical, 45, Article ID: 505303. https://doi.org/10.1088/1751-8113/45/50/505302

[32] Chou, C.-C., Markovich, T. and Kouri, D.J. (2012) Adiabatic Switching Approach to Multidimensional Supersymmetric Quantum Mechanics for Several Excited States. Molecular Physics, 110, 2977-2986. https://doi.org/10.1080/00268976.2012.689874

[33] Williams, C.L., Bodmann, B.G. and Kouri, D.J. (2017) Fourier and Beyond: Invariance Properties of a Family of Integral Transforms. Journal of Fourier Analysis and Applications, 23, 660-678. https://doi.org/10.1007/s00041-016-9482-x

[34] Williams, C.L., Pandya, N., Bodmann, B.G. and. Kouri, D.J. (2017) Coupled Supersymmetric Quantum Mechanics and Ladder Structures beyond the Harmonic Oscillator. Journal of Mathematical Physics, submitted.

[35] Hoffman, D.K. and Kouri, D.J. (1992) Distributed Approximating Functional Theory: A General Approach to Fully Quantal Applications to Wave Propagation. The Journal of Chemical Physics, 96, 1179-1184.

https://doi.org/10.1021/j100182a030

[36] Zhang, D.S., Wei, G.W. and Kouri, D.J. (1997) Distributed Approximating Functional Approach to the Fokker-Planck Equation: Eigenfunction Expansion. The Journal of Chemical Physics, 106, 5216. https://doi.org/10.1063/1.473520

[37] Wei, G.W., Althorpe, S.C., Kouri, D.J. and Hoffman, D.K. (1998) An Application of DAF-Wavelets to Reactive Scattering. The Journal of Chemical Physics, 108, 7065. https://doi.org/10.1063/1.476125

[38] Wei, G.W., Zhang, D.S., Althorpe, S.C., Kouri, D.J. and Hoffman, D.K. (1998) Wavelet-Distributed Approximating Functional Method for Solving the NavierStokes Equation. Computer Physics Communications, 115, 18-24.

[39] Pindza, E. and Moré, E. (2014) Solving Generalized Long Wave Equations Using a 
Distributed Approximating Functional Method. International Journal of Computer Mathematics., 2014, Article ID: 178024.

[40] Maji, K. (2016) Hermite-Distributed Approximating Functional-Based Formulation of Multiconfiguration Time-Dependent Hartree Method: A Case Study of Quantum Tunnelling in a Coupled Double-Well System. Pramana, 87, 34. https://doi.org/10.1007/s12043-016-1243-5

[41] Yao, J., Zhu, T., Hussain, F. and Kouri, D.J. (2016) Solving Fractional Laplacian Viscoacoustic Wave Equation Using Hermite Distributed Approximating Functional Method. SEG Technical Program Expanded Abstracts 2016, 3966-3971.

Submit or recommend next manuscript to SCIRP and we will provide best service for you:

Accepting pre-submission inquiries through Email, Facebook, LinkedIn, Twitter, etc. A wide selection of journals (inclusive of 9 subjects, more than 200 journals) Providing 24-hour high-quality service User-friendly online submission system Fair and swift peer-review system Efficient typesetting and proofreading procedure Display of the result of downloads and visits, as well as the number of cited articles Maximum dissemination of your research work

Submit your manuscript at: http://papersubmission.scirp.org/

Or contact am@scirp.org 